\title{
Construção de um ambiente de Business Intelligence para Secretaria de Assistência Social da Prefeitura Municipal de São Paulo
}

\author{
Construction of a Business Intelligence environment for the Secretariat of Social Assistance of the \\ Municipality of São Paulo \\ Construcción de un ambiente de Business Intelligence para la Secretaría de Asistencia Social del \\ Municipio de São Paulo
}

Recebido: 22/07/2021 | Revisado: 30/07/2021 | Aceito: 19/08/2021 | Publicado: 22/08/2021

\author{
José Avelino Placca \\ ORCID: https://orcid.org/0000-0002-2024-5895 \\ Universidade Estadual do Mato Grosso do Sul, Brasil \\ E-mail: placca@gmail.com \\ Priscila Nery de Castro \\ ORCID: https://orcid.org/0000-0003-3610-6248 \\ Visual Sciense Corp, Brasil \\ E-mail: priscila.castro@visualscience.com.br
}

\begin{abstract}
Resumo
O campo de Ciência dos Dados tem se tornado um dos mercados mais promissores na área de tecnologia nos últimos anos. A utilização de técnicas sofisticadas e de recursos de hardware e software para tratar grandes volumes de dados e prover ambientes integrados para auxiliar na tomada de decisão em grandes e médias organizações tem avançado suas fronteiras e atingido diversos outros setores (públicos e privados) que antes se restringiam apenas ao aspecto transacional dos bancos de dados. Este trabalho tem por objetivo descrever a concepção, modelagem e implementação de um ambiente de gestão de informações da Secretaria Municipal de Assistência e Desenvolvimento Social SMADS da Prefeitura Municipal de São Paulo, visando a modernização da gestão de informações da Secretaria, qualificando a elaboração, implementação e monitoramento das políticas públicas de Assistência Social executadas na cidade de São Paulo. A metodologia utilizada foi a abordagem bottom-up inicialmente apresentada por Ralph Kimball como uma proposta de desenvolvimento de um projeto de DW compreensível e rápido. A criação do DW seguiu as etapas do Processo de KDD: seleção dos dados; pré-processamento; transformação; interpretação e análise. O projeto foi executado através da parceria da Prefeitura de São Paulo com a UNESCO e contemplou todas as etapas de um processo de Business Intelligence (BI). O presente trabalho descreve as etapas de: identificação das necessidades, implantação da infraestrutura operacional, construção do Data Warehouse (DW), implementação dos processos de ETL, elaboração de painéis, elaboração da documentação e treinamento dos diversos perfis de usuários. Foram utilizadas como fontes de dados: base de dados dos sistemas legados utilizados pela SMADS assim como diversas bases externas com destaque para o CadÚnico do Governo Federal. Os resultados contemplaram a criação de um ambiente de Inteligência de Negócios e a disseminação da cultura de criação de modelos de visualização de dados para o processo de tomada de decisão no âmbito da Secretaria de Assistência Social da Prefeitura Municipal de São Paulo que permitiram uma melhor eficácia na aplicação e monitoramento das políticas públicas de assistência social na cidade de São Paulo.
\end{abstract}

Palavras-chave: Business Intelligence; Data Warehouse; KDD process.

\begin{abstract}
The Data Science field has become one of the most promising markets in the technology field in recent years. The use of sophisticated techniques and hardware and software resources to handle large volumes of data and provide integrated environments to assist in decision making in large and medium organizations has advanced its frontiers and reached several other sectors (public and private) that were previously restricted only to the transactional aspect of the databases. This paper aims to describe the conception, modeling and implementation of an information management environment of the Municipal Secretariat for Social Assistance and Development - SMADS of the Municipality of São Paulo, aiming at the modernization of the Secretariat's information management, qualifying the elaboration, implementation and monitoring of public social assistance policies implemented in the city of São Paulo. The methodology used was the bottom-up approach initially presented by Ralph Kimball as a proposal to develop a fast and understandable DW project. The creation of the DW followed the steps of the KDD Process: data selection; preprocessing; transformation; interpretation and analysis. The project was carried out through a partnership between the
\end{abstract}


City of São Paulo and UNESCO and covered all stages of a Business Intelligence (BI) process. This work describes the steps of: identification of needs, implementation of operational infrastructure, construction of the Data Warehouse (DW), implementation of ETL processes, preparation of panels, preparation of documentation and training of different user profiles. The following data sources were used: database of legacy systems used by SMADS as well as several external databases with emphasis on the Federal Government's CadÚnico. The results contemplated the creation of a Business Intelligence environment and the dissemination of the culture of creation of data visualization models for the decision-making process within the scope of the Social Assistance Secretariat of the Municipality of São Paulo, which allowed a better efficiency in the application and monitoring of public social assistance policies in the city of São Paulo.

Keywords: Business Intelligence; Data Warehouse; KDD process.

\section{Resumen}

El campo de la ciencia de datos se ha convertido en uno de los mercados más prometedores en el campo de la tecnología en los últimos años. El uso de técnicas sofisticadas y recursos de hardware y software para manejar grandes volúmenes de datos y proporcionar entornos integrados para ayudar en la toma de decisiones en organizaciones grandes y medianas ha avanzado sus fronteras y ha llegado a varios otros sectores (públicos y privados) que anteriormente estaban restringidos solo a el aspecto transaccional de las bases de datos. Este trabajo tiene como objetivo describir la concepción, modelación e implementación de un ambiente de gestión de información de la Secretaría Municipal de Asistencia Social y Desarrollo - SMADS del Municipio de São Paulo, con el objetivo de modernizar la gestión de información de la Secretaría, calificando la elaboración, implementación y seguimiento de las políticas públicas de asistencia social implementadas en la ciudad de São Paulo. La metodología utilizada fue el enfoque ascendente presentado inicialmente por Ralph Kimball como una propuesta para desarrollar un proyecto de DW rápido y comprensible. La creación del DW siguió los pasos del Proceso KDD: selección de datos; preprocesamiento; transformación; interpretación y análisis. El proyecto se ejecutó a través de una asociación entre la Ciudad de São Paulo y la UNESCO y cubrió todas las etapas de un proceso de Business Intelligence (BI). Este trabajo describe los pasos de: identificación de necesidades, implementación de infraestructura operativa, construcción del Data Warehouse (DW), implementación de procesos ETL, preparación de paneles, preparación de documentación y capacitación de diferentes perfiles de usuarios. Se utilizaron las siguientes fuentes de datos: base de datos de sistemas heredados utilizados por SMADS así como varias bases de datos externas con énfasis en el CadÚnico del Gobierno Federal. Los resultados contemplaron la creación de un ambiente de Business Intelligence y la difusión de la cultura de creación de modelos de visualización de datos para el proceso de toma de decisiones en el ámbito de la Secretaría de Asistencia Social del Municipio de São Paulo, lo que permitió una mejor eficiencia en el aplicación y seguimiento de las políticas públicas de asistencia social en la ciudad de São Paulo.

Palabras clave: Inteligencia de Negocios; Almacén de Datos; Proceso KDD.

\section{Introdução}

Os setores públicos e privados têm sido forçados a tomarem decisões cada vez mais rápidas e precisas. Apesar disso, os sistemas utilizados em suas operações comuns não são voltados para auxiliar no processo de tomada de decisão. O DW surgiu tendo este objetivo.

Organizações de todas as áreas sabem bem da importância de suas decisões estratégicas. Com o nível de concorrência atual, empresas dos mais variados tipos têm sentido uma necessidade cada vez maior de reunir informações confiáveis sobre suas operações e sobre o mercado a fim de tomar ações cada vez mais acertadas de negócios, possibilitando um aumento de lucratividade e de competitividade.

Cenário semelhante ocorre com a Administração Pública. A população tem demandado cada vez mais serviços e de maior qualidade. Dessa forma, a administração pública deve sempre priorizar a execução de serviços com eficiência, obedecendo aos princípios administrativos e fazendo uso otimizado do orçamento público. Para tal, é necessário sistematizar informações sobre o cidadão e suas necessidades para tomar as melhores decisões e evitar desperdício de recursos públicos.

Com o nível atual de automatização, imensas quantidades de dados sobre as operações diárias das organizações passam por sistemas computacionais. Esses sistemas são amplamente utilizados por várias áreas, cada qual com um ou mais sistemas específicos para atender a suas necessidades. Apesar dessa imensa quantidade de dados, os sistemas utilizados no dia a dia não são projetados para permitir a análise desses dados por estrategistas e tomadores de decisão. Muitas vezes, é 
impossível realizar qualquer análise sobre esses dados sem ter de parar a produção ou diminuí-la significativamente.

Além disso, para que se faça uma análise mais qualificada da informação, é necessário cruzar dados de diferentes sistemas.

Dentre as dificuldades já citadas, existe o problema da consistência dos dados entre os diferentes sistemas: como está registrado o cliente/cidadão em cada uma das bases dos diferentes sistemas? O ambiente operacional é voltado para a produção diária, o que também dificulta a tarefa dos tomadores de decisão. As tarefas do dia a dia são facilitadas neste ambiente, que foi projetado para tal, mas consultas mais complexas demandam uma quantidade de tempo e recursos que inviabilizam sua aplicação.

Surge, assim, a necessidade de um ambiente voltado para os tomadores de decisão, que permita que estes analisem dados confiáveis de forma eficiente e eficaz, aumentando a competitividade e a lucratividade de empresas, e o ganho de eficiência e produtividade do setor público.

O objetivo deste trabalho é descrever o projeto de concepção, modelagem e implementação de um ambiente de gestão de informações da Secretaria Municipal de Assistência e Desenvolvimento Social - SMADS da Prefeitura Municipal de São Paulo, visando a modernização da gestão de informações da Secretaria, qualificando a elaboração, implementação e monitoramento das políticas públicas de Assistência Social executadas na cidade de São Paulo. O ambiente foi concebido na forma de centralizar num Data Warehouse todo o repositório das informações dos diversos sistemas de acompanhamento e monitoramento dos programas de assistência social realizados pela SMADS assim como de bases de dados externas relacionadas a políticas de assistência social, permitindo, dessa maneira, a gestão das informações através de ferramentas de geração de relatórios, painéis, cubos, etc.

O projeto é apresentado detalhadamente, mostrando o contexto e o escopo, a metodologia e a estratégia adotada, as ferramentas utilizadas, e as soluções adotadas para os principais desafios, bem como os resultados obtidos.

\section{Metodologia}

Devido à estrutura organizacional da SMADS, a diversidade de sistemas utilizados pelos diversos órgãos e instituições que a compõem, bem como a estratégia adotada de envolvimento das pessoas responsáveis nas etapas inicias do projeto de construção do DW optou-se por adotar a metodologia proposta por (Kimball, 1996) também conhecida como abordagem bottom-up que começa com a extração, transformação e a integração dos dados para um ou mais DMs, sendo estes DMs modelados, normalmente através de um modelo dimensional (Elias, 2014). Devido essas particularidades a abordagem utilizada foi o método qualitativo (Estrela, 2018) onde seguiu-se o roteiro tradicional para a construção de DW.

\section{Estrutura da SMADS}

A Secretaria Municipal de Assistência e Desenvolvimento Social (SMADS) possui como objetivo formular, coordenar, implementar, executar, monitorar e avaliar políticas e estratégias para o Sistema Único de Assistência Social SUAS no âmbito do Município de São Paulo. Compete a ela funções de proteção, defesa e vigilância sociais, considerando as disposições, normativas da Lei Orgânica de Assistência Social (LOAS), da Norma Operacional Básica do SUAS.

\section{Política de Assistência Social}

O município de São Paulo encontra-se habilitado em nível de gestão plena, ou seja, tem a responsabilidade total das ações da assistência social. A Secretaria possui três principais recursos de gestão. São eles: o Plano Municipal de Assistência Social (PLAS), o Conselho Municipal de Assistência Social (COMAS) e o Fundo Municipal de Assistência Social (FMAS). 
Tais recursos possuem políticas-fim, que têm o intuito de auxiliar no atendimento integral de famílias, crianças e adolescentes, mulheres, idosos, pessoas em situação de rua e pessoas com deficiência que por algum motivo se encontram em situação de maior vulnerabilidade social. Dessa forma a Secretaria responde pela concessão, gestão ou orientação às famílias quanto aos benefícios socioassistenciais que são de três modalidades:

- Continuados (transferência direta e regular de renda): Benefício de Prestação Continuada (BPC) para pessoas idosas e pessoas com deficiência (federal), o Bolsa Família (federal), o Renda Cidadã (estadual), o Ação Jovem (estadual) e a garantia de Renda Mínima (municipal);

- Eventuais: Suplementação alimentar, bilhete de transporte urbano, segunda via de documentos pessoais, passagens rodoviárias intermunicipais e interestaduais, fotos e fotocópias para documentos pessoais, entre outros;

- Emergenciais: Abrigo e suprimentos alimentares e de higiene.

Já as políticas-meio são de convênios, recursos humanos, comunicação institucional, relações interinstitucionais, tecnologia da informação, vigilância social, gestão do espaço físico, instalações e equipamentos e gestão de recursos financeiros. Há ainda os recursos federais que não integram o orçamento municipal e que, no entanto, destinam-se à área de Assistência Social na cidade de São Paulo, como são os casos do Bolsa Família e do Benefício de Prestação Continuada, em que os valores são repassados diretamente aos beneficiários.

Os serviços da rede indireta são ofertados mediante convênios com organizações sociais com capacidade para atender cerca de 220 mil pessoas, registrando assim, uma das maiores redes de serviços socioassistenciais da América Latina. Esses serviços são subdivididos em Serviços de Proteção Social Básica, Serviços de Proteção Social Especial de Média Complexidade e Serviços de Proteção Social Especial de Alta Complexidade. Quanto aos serviços de rede direta, destacam-se os Centros de Referência de Assistência Social (CRAS) destinados à prevenção de situações de vulnerabilidade e risco social nos territórios por meio do fortalecimento de vínculos familiares e comunitários, os Centros de Referência Especializados de Assistência Social (CREAS), que oferecem serviços especializados e continuados a famílias e indivíduos nas diversas situações de violência e violação de direitos, e os Centro de Referência Especializados para População em Situação de Rua (Centro POP) voltados para o atendimento especializado à população em situação de rua.

\section{Vigilância Socioassistencial}

A Coordenação do Observatório da Vigilância Socioassistencial (COVS) é o setor da SMADS responsável pela função de vigilância socioassistencial da política de assistência social da Cidade de São Paulo, produzindo, sistematizando e analisando informações territorializadas sobre as situações de vulnerabilidade e risco que incidem sobre famílias e indivíduo e sobre os padrões de oferta dos serviços e benefícios socioassistenciais, considerando questões correlatas ao padrão de financiamento, ao tipo, volume, localização e qualidade das ofertas e das respectivas condições de acesso. Tais informações subsidiam a SMADS com a elaboração de diversos diagnósticos, estudos e pesquisas de vigilância socioassistencial, apoiando a gestão na tomada de decisões quanto à oferta e qualidade dos serviços da rede socioassistencial.

Nesse contexto, a SMADS celebrou o Projeto 914BRZ3019 em parceria com a UNESCO para a contratação de consultoria especializada em revisão de rotinas e modernização da gestão de informações, de modo a qualificar a elaboração, implementação e monitoramento das políticas públicas de Assistência Social, realizados precipuamente por meio de planilhas.

Para tanto, o primeiro produto do projeto entregue pela consultoria foi um plano de trabalho, que contemplava as diversas fases de um projeto de implantação de um ambiente de gestão da informação na SMADS. 
O objetivo principal do referido projeto foi de proporcionar um ambiente informacional que congregasse diversas fontes de dados de interesse da SMADS para subsidiar as análises técnicas sobre os diversos serviços assistenciais prestados pela Prefeitura Municipal de São Paulo bem como o processo de tomada de decisão pertinente a gestão da cadeia de atendimento social composta por: 1230 serviços da Rede Indireta (convênios) e 90 serviços da Rede Direta (54 CRAS, 30 CREAS e 6 CENTROS POP) (Raio-X,2021)

\section{Sistema de Tomada de Decisão}

Um Sistema de Tomada de Decisão utilizando-se dos conceitos e ferramentas de BI pressupõe a existência de um ambiente de processo único e confiável que recebe dados, passa por um processo de tratamento e limpeza, e é disponibilizado em formato de fácil entendimento para a análise e tomada de decisão (Inmon, 1997). Neste Sistema, se tem a certeza de que a informação disposta nele está correta e com qualidade validada, uma vez que passou por todos os processos e crivos para estar lá (Costa, Silva, 1999).

Figura 1 - O processo de Inteligência de Dados - SMADS.

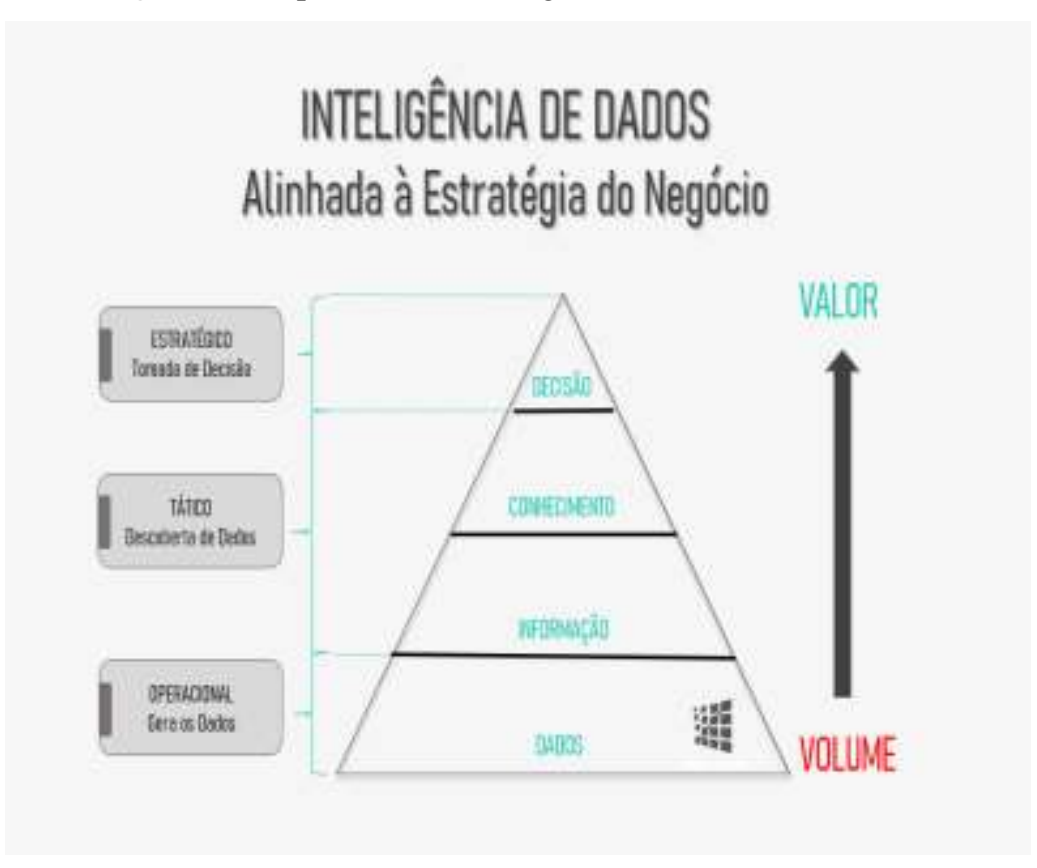

Fonte: Autores.

\section{Definição dos processos de OLTP e OLAP}

A sigla OLTP (Online Transaction Processing ou Processamento de Transações em Tempo Real) refere-se aos sistemas transacionais (softwares e aplicativos), aqueles sistemas que são lançadas as informações diariamente. Seu objetivo é coletar e processar os dados de rotina e dar apoio à execução do negócio. Alguns exemplos: SISA, SISRUA, SISCR, etc.

A sigla OLAP (Online Analytical Processing ou Processamento Analítico em Tempo Real) é o termo usado para se referir à capacidade de analisar informações. É a interface de análise de dados que foram extraídos por outros sistemas. OLAP se concentra na tomada de decisão, seu objetivo é a transformação dos dados em informação e conhecimento, para tomada de decisão (Chaudhuri, Dayal, 1997). Diferente do OLTP, o OLAP não é um software único, são ferramentas/softwares de análises que juntos, cada um com sua característica, formam um "esquema" de análise de dados dando vida ao sistema de apoio à tomada de decisão (Berson \& Smith 1997). Alguns exemplos: ETL+DW+Dashboards, cubos analíticos, etc.

De forma resumida, armazenamos e atualizamos registros de dados usando OLTP, e analisamos usando OLAP. 
Figura 2 - Diferenciação entre processos OLTP X OLAP.

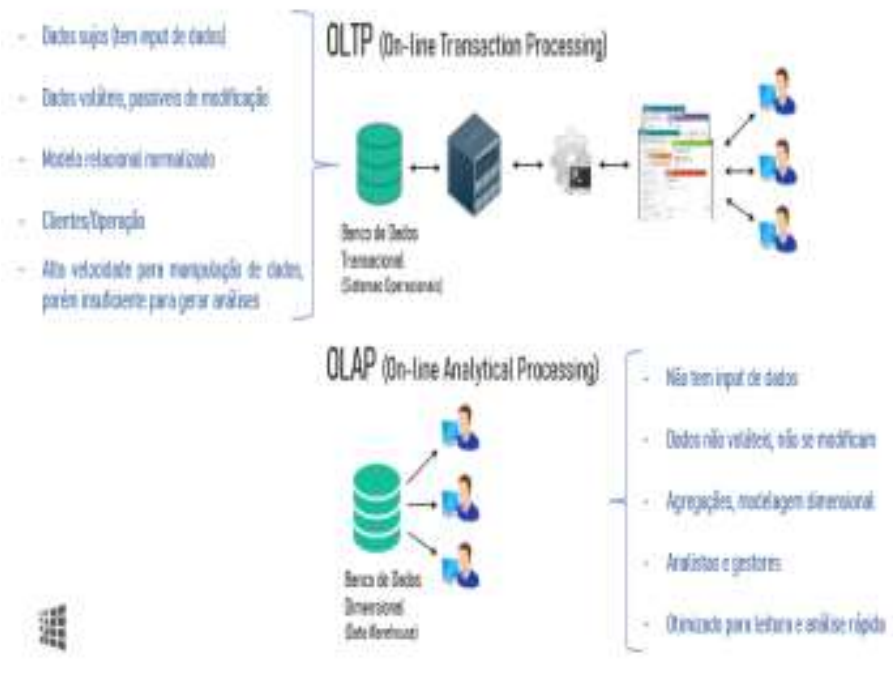

Fonte: Autores.

\section{Etapas do Projeto DW-SMADS}

O projeto foi realizado em etapas identificadas pelos 12(doze) processos indicados conforme o esquema abaixo:

Figura 3 - Etapas do Processo de Implantação do DW-SMADS.

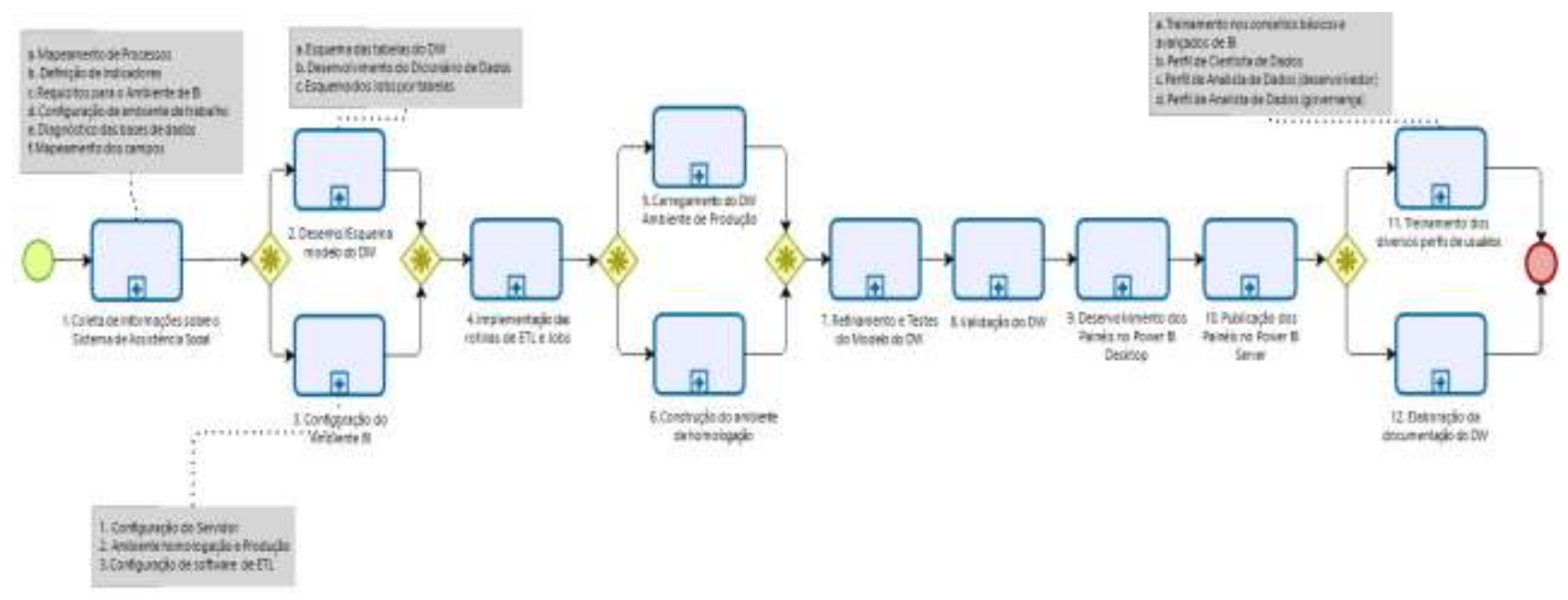

Fonte: Autores.

1) Coleta de informações sobre o Sistema de Assistência Social

2) Desenho/esquema do Data Warehouse (DW)

3) Configuração do ambiente de Business Intelligence (BI)

4) Implementação das rotinas de ETL e Jobs

5) Carregamento do DW no ambiente de produção

6) Construção do ambiente de homologação

7) Refinamento e testes do modelo do DW

8) Validação do DW

9) Desenvolvimento dos painéis no Power BI

10) Publicação dos painéis no Power BI Server

11) Treinamento dos diversos perfis de usuários

12) Elaboração da documentação do DW 


\section{Configuração do Ambiente}

Foram levantados os requisitos tecnológicos necessários para a execução das atividades planejadas. A configuração abaixo foi estruturada com base nas possibilidades de infraestrutura disponíveis para a SMADS no momento da implantação.

Figura 4 - Metodologia e estrutura tecnológica para o Sistema de Apoio à Decisão.

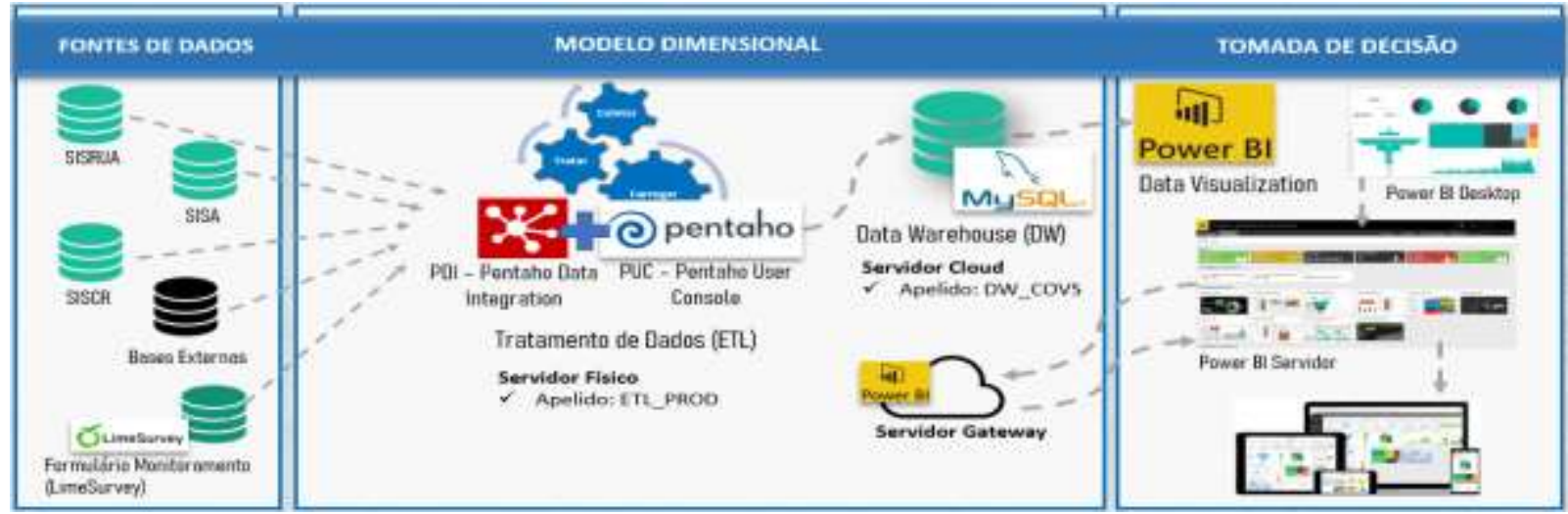

Fonte: Autores.

A solução de BI foi dividida em três partes:

$1^{\circ}$ ) Servidor de ETL (Extract, Transform and Load):

ETL no contexto de infraestrutura significa a máquina que vai executar o processo de extração, transformação e carga das fontes de dados para dentro do Data Warehouse (DW).

$2^{\circ}$ ) Servidor de Armazenamento de Dados - Data Warehouse:

DW no contexto da arquitetura de BI significa a máquina que hospeda o banco de dados - hardware e software - que vai cumprir a função de armazenar os dados para o consumo da solução de visualização de dados. Para definir esse componente do processo é necessário conhecer: o volume de dados que será carregado inicialmente, e sua previsão de crescimento (em bytes ou registros por mês), e a quantidade de usuários que realizarão consultas. Estas informações não são disponíveis prontamente, normalmente é realizada uma estimativa.

Um DW não deve ser considerado para oferecer consumo direto a nenhum sistema OLTP. O DW é um componente do processo de BI somente.

$3^{\circ}$ ) Servidor de Exploração de Dados:

Uma vez que os dados estejam disponíveis no DW, os usuários começam a acessá-los e a explorá-los, dentro de um ambiente OLAP para resolver suas diversas necessidades de análise. Aqui o resultado final são os dashboards.

\section{Seleção dos Dados nas Fontes de Origem}

Inicialmente foi realizado o levantamento e Diagnóstico das bases de dados disponíveis na SMADS

As bases de dados da SMADS se compõem de um conjunto de base de dados (SQL Server) de sistemas da SMADS e de um conjunto de bases em Excel (DEMES e FORMS) e de um conjunto de bases externas em Excel e Arquivo-Texto descritas a seguir: 


\section{$\underline{\text { Bases Internas (sistemas SMADS) }}$}

As bases internas são compostas pelas seguintes bases de dados:

- $\quad$ SISA (SQL Server)

- $\quad$ SISCR/BDC (SQL Server)

- $\quad$ SISRUA (SQL Server)

\section{Bases Internas (DEMES e FORMS)}

Do período de junho de 2013 a abril de 2020 a SMADS vem utilizando a DEMEs (Demonstrativo de Execução Mensal) para capturar e analisar indicadores da prefeitura na área de Assistência Social.

Até abril de 2020 havia no total 52 modelos de DEMEs, sendo 27 da alta proteção, 14 da média e 11 da básica.

Devido a falta de padronização das informações contidas nas DEMES não foi possível fazer a carga das informações para o DW.

Foi utilizado o Pentaho ${ }^{\circledR}$ como ferramenta de ETL. Para cada arquivo de carga de tabelas foram implementadas as devidas transformações para o modelo final no DW.

\section{Extração, Transformação e Carregamento}

Esta etapa consiste na carga do modelo dimensional.

Os processos de carga são realizados por modelos dimensionais. Utilizando o servidor de ETL realizamos a conexão com as fontes de dados e a extração dos dados de interesse. Estes dados passam por um fluxo de transformação onde é realizada a limpeza e a inclusão das variáveis de negócio. Na sequência, o dado é carregado no DW em tabelas conforme o modelo dimensional previamente estruturado.

\section{Modelo do DW-SMADS}

O DW foi projetado em função da origem dos dados coletados resultando em 3 DMs (Data Marts) relativos aos sistemas SISA, SISCR e SISRUA utilizando-se das informações das bases de dados externas, particularmente o CadÚnico. 
Research, Society and Development, v. 10, n. 11, e16101118577, 2021

(CC BY 4.0) | ISSN 2525-3409 | DOI: http://dx.doi.org/10.33448/rsd-v10i11.18577

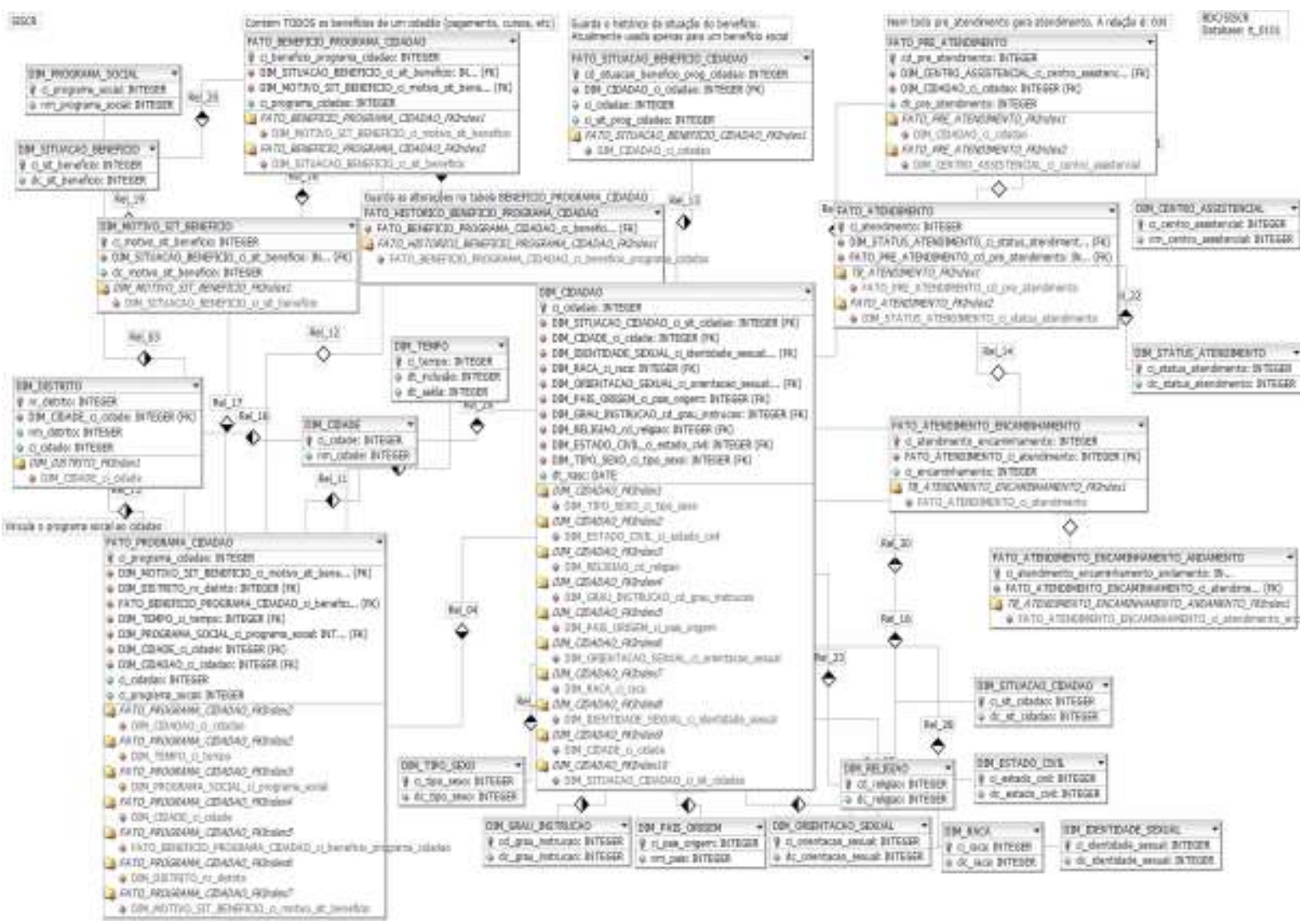

Fonte: Autores.

Figura 6 - Data Mart 02 - SISA.

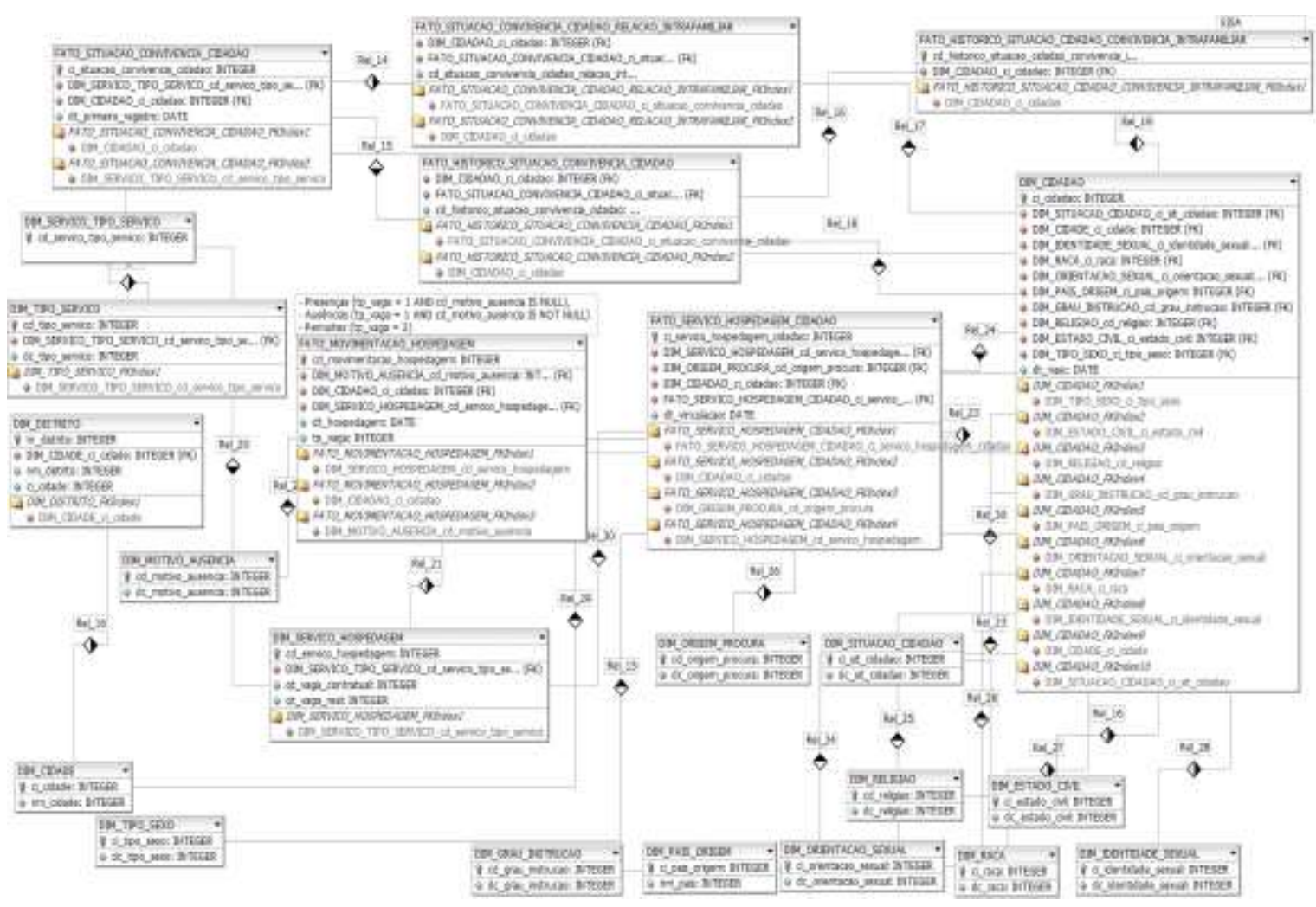

Fonte: Autores. 
Figura 7 - Data Mart 03 - SISRUA.

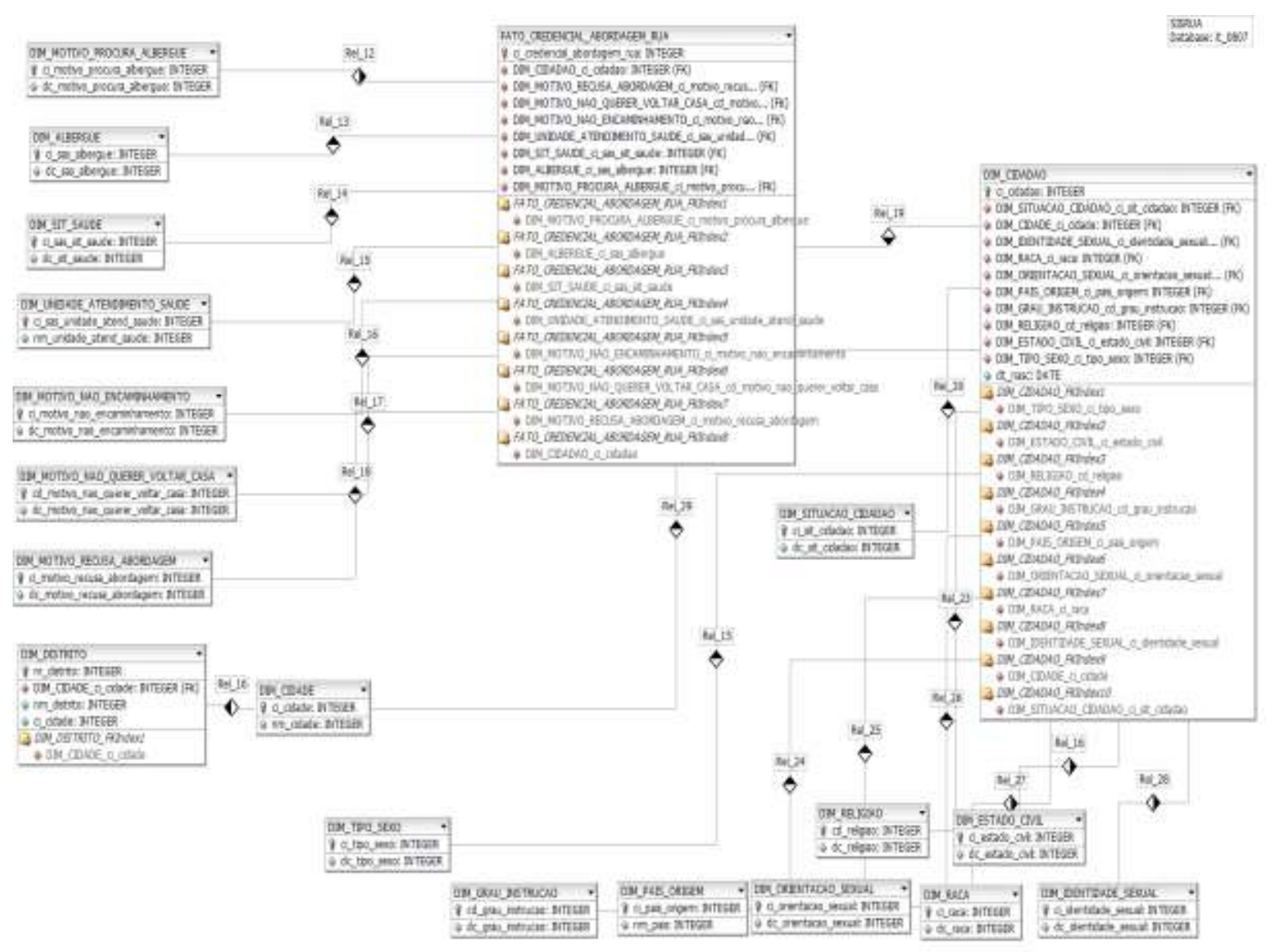

Fonte: Autores.

Para a construção do DW e de cada Data Mart foi elaborada uma matriz de necessidades com base no que era necessário ser incorporado ao DW, através de um processo de levantamento e identificação dos indicadores. Na matriz foram acrescentados todos os requisitos do DW e relacionado com as métricas e seus respectivos descritores (Kimball \& Ross, 2002), (Machado, 2007).

\section{Resultados e Discussão}

\section{Construção de Dashboards}

Foi utilizado no desenvolvimento dos dashboards, o software Power BI Desktop, para posterior publicação e acesso no Power BI Server com atualização diária. Os dados de consumo são originados do Data Warehouse (DW) da COVS, já utilizando as tabelas de fato e dimensões tratadas e preparadas para uso da COVS em suas análises.

No conjunto de produtos entregues, foram elaborados dashboards de gestão e monitoramento das redes direta e indireta, alguns serão citados:

\section{- Central de vagas}

Elaborado com o objetivo de monitorar em tempo real a disponibilidade de vagas nos serviços de acolhimento da rede socioassistencial para permitir encaminhamento dos cidadãos e cidadãs de forma mais simples e ágil.

A SMADS está estruturando a criação de uma mesa da Central de Vagas no $1^{\circ}$ semestre de 2021 na CPAS 
(Coordenação de Pronto Atendimento Social), setor responsável pelas solicitações de atendimento a pessoas em situação de rua e também pela central de vagas de acolhimento para adultos, crianças e adolescentes.

Atualmente já existe um boletim informativo diário no formato de planilha de Excel que informa a disponibilidade das vagas da rede socioassistencial parceira. Também há um relatório dentro do SISA (Sistema de Informação do Atendimento aos Usuários) capaz de informar a disponibilidade das vagas dos serviços de forma tipificada (vagas masculinas, femininas, vagas acessíveis e não acessíveis, quantidade de berços e quartos para famílias). Entretanto, tanto o boletim quanto o relatório de vagas são limitados e satisfazem plenamente os objetivos da Central de vagas por serem estáticos, não mostrando a disponibilidade de vagas mais recente e não permitindo a utilização de filtros dinâmicos.

Como a mesa da Central de Vagas precisa da informação da disponibilidade de vagas em tempo real, a Central acaba realizando ligações telefônicas diretamente para os serviços, despendendo muito tempo para saber o status da disponibilidade das vagas.

Nesse contexto, o painel visa então facilitar a gestão das vagas da rede socioassistencial, permitindo que os colaboradores da Central de Vagas visualizem a disponibilidade das vagas por gênero, acessibilidade, SAS, distrito, tipologia, serviço, público-alvo, segmento. Por fim, o painel permite ainda visualizar a disponibilidade de berços e quartos para famílias.

Figura 8 - Dashboard da Central de Vagas.

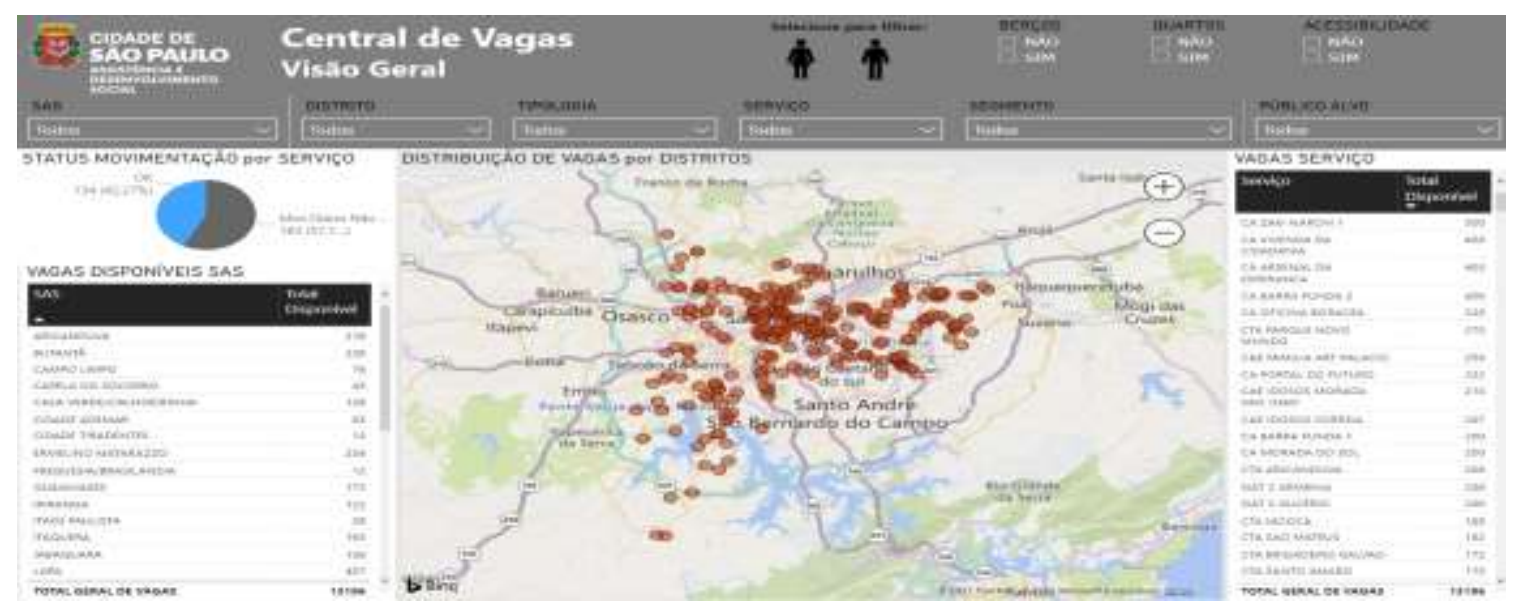

Fonte: Autores.

\section{- Medida protetiva - informações de acolhidos}

Elaborado com o objetivo de monitorar o preenchimento dos campos necessários ao atendimento de acolhidos de medidas protetivas. Serão monitorados dois indicadores: 1) \% Acolhidos com informação incompleta 2) \% não de preenchimento de cada campo. A partir desses indicadores os serviços serão orientados a preencher corretamente o cadastro do acolhido,

Acompanhar os dados de medidas protetivas de crianças e adolescentes dos serviços de SAICA, Casa Lar e Família Acolhedora é imprescindível para cessar a situação de risco, proteger a criança ou adolescente e garantir o pleno gozo dos direitos ameaçados ou violados.

Os dados mais importantes de medidas protetivas dizem respeito ao Distrito e SAS de Origem do acolhido, data do processo, vara de infância, destituição do poder familiar, existência de irmãos em serviços de acolhimento, perspectiva de reinserção familiar e prazo, acompanhamento PPCAM, ameaça de morte e proposta de reordenamento com a sua devida justificativa.

Tais dados são atualmente inseridos no Sistema SISA (Sistema de Informação do Atendimento aos Usuários) pela 
rede socioassistencial parceira. O Observatório da Vigilância Socioassistencial se empenha para cobrar tal preenchimento e capacitar a rede parceira para que esses dados sejam atualizados sempre que necessário, pois impactam diretamente a vida da criança e do adolescente acolhido.

Atualmente, o preenchimento dos dados das medidas protetivas é monitorado mensalmente e forma bastante operacional, envolvendo a extração de dados brutos, manipulação e tratamento de planilhas de Excel. Nesse contexto, o painel permitirá visualizar diariamente a quantidade de crianças e adolescentes que estão com informações das medidas protetivas incompletas.

Como consequência, a expectativa é que haja maior preenchimento e atualização das informações das medidas protetivas, aumentando a possibilidade de reordenamento das vagas da rede socioassistencial por meio de dados de georreferenciamento de onde são (território de origem) e onde estão acolhidas as crianças e adolescentes.

Figura 9 - Dashboard da Medidas Protetivas.

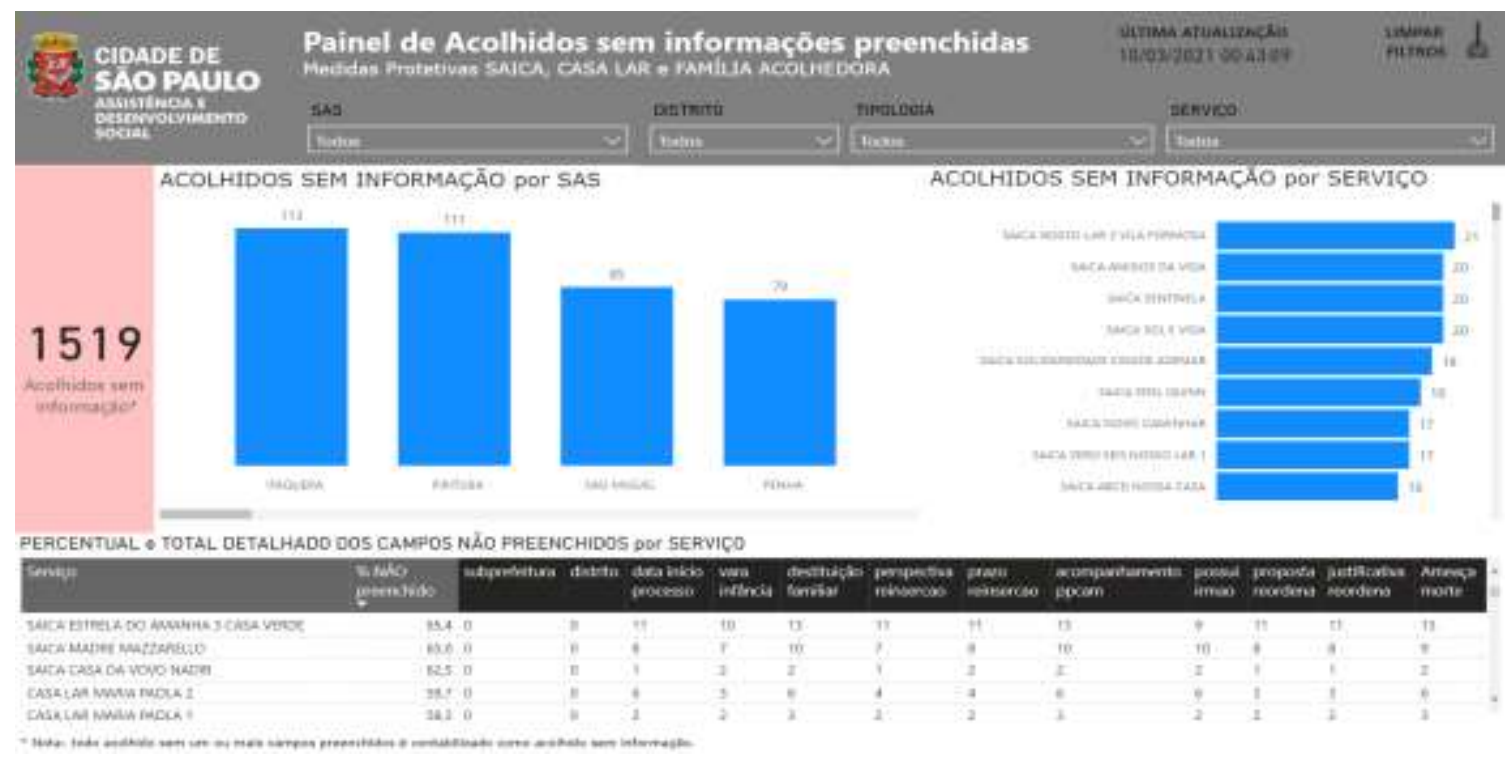

Fonte: Autores.

\section{- Gestão SMADS - Perfil da população acolhida}

Elaborado com o objetivo de possibilitar que os gestores da SMADS possam identificar características globais dos cidadãos atendidos pelos programas assistenciais.

Atendimento às orientações da NOB/SUAS/2012 especialmente:

A Vigilância Socioassistencial deverá cumprir seus objetivos, fornecendo informações estruturadas que:

I. contribuam para que as equipes dos serviços socioassistenciais avaliem sua própria atuação;

II. ampliem o conhecimento das equipes dos serviços socioassistenciais sobre as características da população e do território de forma a melhor atender às necessidades e demandas existentes;

III. proporcionem o planejamento e a execução das ações de busca ativa que assegurem a oferta de serviços e benefícios às famílias e indivíduos mais vulneráveis, superando a atuação pautada exclusivamente pela demanda espontânea. 
Figura 10 - Dashboard de perfil da população acolhida.

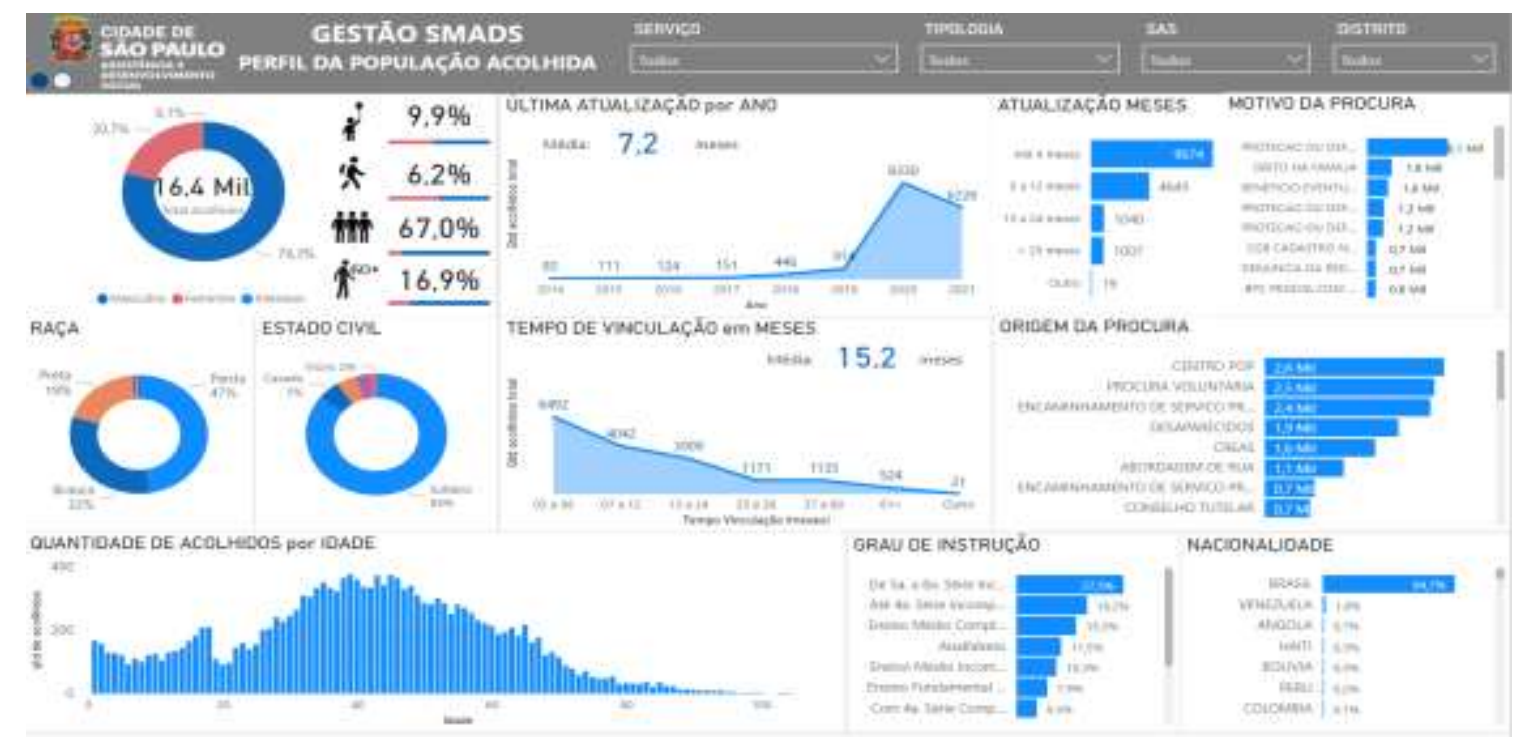

Fonte: Autores.

Os dashboards foram construídos seguindo as boas práticas para a elaboração de painéis no sentido de se "contar uma história" sobre determinado assunto (Knafic, 2017).

\section{Ferramentas Utilizadas}

Foram utilizadas as seguintes ferramentas no processo de construção do ambiente de BI: Pentaho ${ }^{\circledR}$ (ETL), Banco de Dados MySQL @ (DW), Power BI ® (construção, análise e visualização de dashboards).

\section{Considerações Finais}

O projeto de implantação de um ambiente de gestão e monitoramento de informações na SMADS através do patrocínio da UNESCO contou com o trabalho conjunto dos consultores UNESCO e equipe da Coordenação da Vigilância Sanitária da Secretaria de Assistência Social da Prefeitura de São Paulo. O projeto se desenvolveu buscando soluções tecnológicas e de infraestrutura que estivessem disponíveis dentro do orçamento da SMADS. Foram priorizadas as ferramentas de software livre num primeiro momento e, em seguida, as ferramentas já disponíveis na Secretaria. A seleção das bases de dados (internas e externas) ocorreu em função de um levantamento das necessidades de toda estrutura da SMADS bem como para o atendimento das instruções normativas que regulam o trabalho de assistência social na cidade de São Paulo. O resultado final do projeto foi a disseminação da metodologia utilizada assim como a inserção da cultura analítica na equipe da COVS. A equipe ficou estruturada e com capacidade para dar continuidade nas atividades iniciadas pelos consultores.

\section{Agradecimentos}

À equipe da Coordenação do Observatório da Vigilância Social da Secretaria de Assistência Social da Prefeitura Municipal de São Paulo pelas sugestões, observações e pronto atendimento das demandas relativas ao presente projeto. À UNESCO pelo apoio e participação no projeto 
Research, Society and Development, v. 10, n. 11, e16101118577, 2021

(CC BY 4.0) | ISSN 2525-3409 | DOI: http://dx.doi.org/10.33448/rsd-v10i11.18577

\section{Referências}

Berson A., \& Smith S. J. (1997). On Line Analytical Processing - Data Warehousing, Data Mining \& OLAP. Computing McGraw Hill

Chaudhuri, S., \& Dayal, U., (1997). An overview of data warehousing and OLAP technology, SIGMOD Rec., 26(1), 65-74

Costa, M. D., \& Silva, I. A. (1999) Da. Inteligência competitiva: uma abordagem sobre a coleta de informações publicadas. Informação \& Sociedade, 9(1).

Elias, D. (2014). A abordagem top-down e bottom-up no Data Warehouse. https://canaltech.com.br/infra/a-abordagem-top-down-e-bottom-up-no-datawarehouse-21108.

Estrela, C. (2018). Metodologia Científica: Ciência, Ensino, Pesquisa. Editora Artes Médicas.

Inmon, W. H. (1997). Como Construir o Data Warehouse. Editora Campus.

Kimball, R. (1996). The data warehouse toolkit: practical techniques for building dimensional data warehouses, John Wiley \& Sons, Inc., 374

Kimball, R., \& Caserta, J. (2004). The Data Warehouse ETL Toolkit: Practical Techniques for Extracting, Cleaning, Conforming, and Delivering Data, John Wiley \& Sons, 491

Kimball, R., Reeves, L., Ross, M., \& Thornwaite, W. (1998). The Data Warehouse Lifecycle Toolkit: Expert Methods for Designing, Developing and Deploying Data Warehouses, John Wiley \& Sons, Inc., 800

Kimball, R., \& Ross, M. (2002). The Data Warehouse Toolkit: The Complete Guide to Dimensional Modeling, John Wiley \& Sons, Inc., 421

Knafic, C. N. (2017). Storytelling com dados: um guia sobre visualização de dados para profissionais de negócios. Alta Books

Machado, F. N. R. (2007). Tecnologia e Projeto de DW. Editora Érica.

Pessato, T.; \& Stein, M. (2014). O design como diferencial estratégico na construção de dashboards. Congresso Brasileiro de Pesquisa e Desenvolvimento em Design..

Raio-X da Rede Socioassistencial do município de São Paulo de janeiro de 2021. https://www.prefeitura.sp.gov.br/cidade/secretarias/assisten cia_social/observ atorio_social/monitoramento/?p=170850

Shams K., \& Farishta M. (2001), Data Warehousing: Toward knowledge. Top. Health Informatics Management. 21(3), 24-32

ZentuT. (2021) Kimball vs Inmon in Data Warehouse Architecture. https://www.zentut.com/data-warehouse/ralph-kimball-data-warehouse-architecture/. 